\title{
Optimal confidence regions for the two-parameter exponential distribution based on records
}

\begin{abstract}
In this article, we propose two families of optimal confidence regions for the location and scale parameters of the two-parameter exponential distribution based on upper records. Constrained optimization problems are used to find the smallest-area confidence regions for the exponential parameters with a specified confidence level. Optimal prediction interval for the future records is also proposed. Two numerical examples as well as a simulation study, are presented for illustrative purposes. It is shown that the reduction in area of the optimal joint confidence region with respect to the existing confidence regions is substantial.
\end{abstract}

Keyword: Constrained optimization; Lagrangian method; Joint confidence regions 